\title{
ESTUDIO DEL PROCESO DE DESARROLLO DE PRODUCTOS EN INDUSTRIAS ALIMENTICIAS
}

Melisa De Greef (melisadegreef@gmail.com) - Departamento de Ingeniería y Gestión Industrial, Facultad de Ingeniería Química, Universidad Nacional del Litoral

Leticia Milena Arcusin (larcusin@fiq.unl.edu.ar) - Departamento de Ingeniería y Gestión Industrial, Facultad de Ingeniería Química, Universidad Nacional del Litoral

Germán Rossetti (groseti@fiq.unl.edu.ar) - Departamento de Ingeniería y Gestión Industrial, Facultad de Ingeniería Química, Universidad Nacional del Litoral

Daniela Ferreira Campos (ferreiracamposdaniela@gmail.com) - Departamento de Ingeniería y Gestión Industrial, Facultad de Ingeniería Química, Universidad Nacional del Litoral

\begin{abstract}
RESUMEN
El proceso de desarrollo de productos (PDP) es la tarea sistemática cuyo propósito es generar nuevos productos o modificacines en los existentes. En el caso de las industrias alimenticias, el PDP reviste una importancia crucial, debido a que el escenario donde se desenvuelven es altamente competitivo. En el presente trabajo se realiza un estudio comparativo de la Gestión del PDP en empresas alimenticias radicadas en la provincia de Santa Fe (Argentina). Para el análisis se seleccionaron once empresas pertenecientes a tres sectores de actividad económica, definidos en función de su importancia para la región: lácteos, insumos y carnes. Se describen las prácticas habituales de las empresas y se procede a compararlas con las propuestas por un Modelo de Referencia para la industria alimenticia. Entre las principales conclusiones se evidencia que solo algunas empresas disponen de metodologías y prácticas internalizadas para la Gestión del PDP. Además, existen diferencias sustanciales en relación a los niveles de detalle que presenta la sistematización de las actividades que involucra el PDP. Estas pueden observarse, fundamentalmente, en relación al tamaño de las organizaciones. Además, la información indica que, a partir de la comparación entre diferentes sectores, el sector carnes presenta menor nivel de desarrollo del PDP.
\end{abstract}

Palabras clave: Desarrollo de Productos, Industrias alimenticias, Estudio comparativo

Área: Modelos de processo para o desenvolvimento de produtos e serviços

\section{INTRODUCION}

El proceso de desarrollo de productos (en adelante, PDP) es la tarea sistemática que tiene como propósito generar nuevos productos, ya sea introduciendo modificaciones en un producto existente o generando otro completamente nuevo y original (LERMA KIRCHNER, 2010). En el caso de las industrias productoras de alimentos, el PDP reviste una importancia crucial, debido a que el escenario donde se desarrollan las empresas del sector se caracteriza por altos niveles de competitividad y una evolución en diversas perspectivas: seguridad alimentaria, sustentabilidad, embalaje, entre otras (ABU, 2012). Gestionar adecuadamente el proceso implica que las empresas apliquen métodos y herramientas específicas, lo que les permite aumentar su eficacia y también sus beneficios.

El objetivo del presente trabajo es realizar un estudio sobre la Gestión del Proceso de Desarrollo de Productos en empresas productoras de alimentos de la Provincia de Santa Fe. 
La investigación se enmarca en un Proyecto orientado a proponer un Modelo de Gestión del PDP para empresas productoras de alimentos de la Provincia de Santa Fe. El trabajo presenta los resultados preliminares correspondientes a once empresas de la Región 3 Nodo Santa Fe, según regionalización definida por el Estado Provincial a partir del año 2008.

\section{METODOLOGIA}

La presente investigación es de carácter exploratorio-descriptivo (SAUTU et al, 2005), y tiene como fin principal realizar un estudio comparativo de las prácticas habituales relacionadas con la Gestión del Proceso de Desarrollo de Productos en empresas productoras de alimentos radicadas en la provincia de Santa Fe (Argentina), pertenecientes a tres sectores de actividad, seleccionados en función de su importancia para la región y definidos en base a la clasificación propuesta para la Industria Manufacturera por un organismo público nacional: lácteos (Elaboración de productos lácteos); insumos (definido a partir de los rubros Preparación de frutas, hortalizas y legumbres y Elaboración de productos alimenticios no clasificados) y carnes (Producción y procesamiento de carne y productos cárnicos). Para el análisis, se describen las prácticas habituales de las empresas bajo estudio y se procede a compararlas con las actividades propuestas por un Modelo de Referencia para la industria de alimentos.

Con el objetivo de conocer con profundidad la forma en que las empresas efectúan la Gestión del PDP, se realiza un relevamiento a través de entrevistas semi-estructuradas y observación in situ en once empresas, denominadas en adelante empresas A, B, C, D (sector lácteo), E, F, $\mathrm{G}, \mathrm{H}$ (sector insumos), I, J y K (sector carnes). Las entrevistas fueron realizadas al personal vinculado con el desarrollo de productos o a la gerencia de la empresa.

\section{MARCO TEORICO}

El desarrollo de productos es un proceso complejo, mediante el cual una organización transforma oportunidades de mercado y las posibilidades técnicas en informaciones para la fabricación de un producto comercial (HOLMAN, 2004). El PDP involucra todas las áreas de la organización y genera una variada cantidad de información. Está compuesto por etapas interdependientes que abarcan desde la identificación de las necesidades de los consumidores hasta el lanzamiento y seguimiento del producto en el mercado (ROZENFELD et al, 2006) Cabe aclarar, siguiendo a Penso (2003), que se entiende por desarrollo de productos tanto a la elaboración de nuevos productos como a la modificación o relanzamiento de productos existentes.

En la literatura existen diferentes propuestas de sistematización de las actividades de desarrollo de productos en modelos de PDP, dependiendo del área de conocimiento de la cual provienen los autores que tratan el tema. Sin embargo, en muchos casos las diferencias entre esas propuestas son más terminológicas que conceptuales. Rozenfeld et al. (2006) presenta la contribución más completa del PDP, separando el proceso en tres macrofases: pre-desarrollo, desarrollo y post-desarrollo. En relación al sector alimenticio, algunos modelos de desarrollo que se destacan son: Graf et al. (1991), Fuller (1994), Earle (1997) y finalmente el modelo de Penso (2003).

El modelo de referencia desarrollado por Penso, que se basa en la metodología propuesta por Rozenfeld, busca realizar mejoras en relación a los modelos hasta entonces publicados, para poder adaptarlos al sector de alimentos. Se encuentra estructurado en tres macrofases, predesarrollo, desarrollo y post-desarrollo, similares a las de Rozenfeld, diferenciándose de éste por adicionar al modelo algunas actividades específicas del área de alimentos en la macrofase 
de desarrollo (en el diseño conceptual: definición de la formulación del producto, procesos de fabricación, embalajes, parámetros y plazos de validación; en el diseño detallado: elaboración del diseño de producto y embalaje, especificación del proceso productivo, del embalaje, de stock y distribución, realización de la revisión del manual Buenas Prácticas de Manufactura, capacitación del personal y elaboración de los manuales de calidad, como APPCC (Análisis de Peligros y Puntos Críticos de Control); en la preparación de la producción: producción del lote piloto y realización de análisis físico-químicos, microbiológicos, sensoriales y test de estabilidad del producto). La Figura 1 presenta el Modelo Referencial propuesto por Penso.

Figura 1. Modelo de Referencia para la Gestión del PDP. Fuente: Penso (2003)

\begin{tabular}{|c|c|c|c|c|c|c|c|c|c|}
\hline \multicolumn{3}{|c|}{ PRE-DESARROLLO } & \multicolumn{5}{|c|}{ DESARROLLO } & \multicolumn{2}{|c|}{ POST-DESARROLLO } \\
\hline $\begin{array}{c}\text { Planificación } \\
\text { estratégica } \\
\text { del producto }\end{array}$ & $\begin{array}{l}\text { Planificación } \\
\text { del Portfolio } \\
\text { de productos }\end{array}$ & $\begin{array}{l}\text { Planificación } \\
\text { de productos }\end{array}$ & $\begin{array}{c}\text { Diseño } \\
\text { informacional }\end{array}$ & $\begin{array}{c}\text { Diseño } \\
\text { conceptual }\end{array}$ & $\begin{array}{r}\text { Diseño } \\
\text { detallado }\end{array}$ & $\begin{array}{c}\text { Preparación } \\
\text { de la } \\
\text { producción }\end{array}$ & | Lanzamiento & $\begin{array}{c}\text { Acompaña- } \\
\text { miento del } \\
\text { producto }\end{array}$ & $\begin{array}{l}\text { Retiro del } \\
\text { producto }\end{array}$ \\
\hline Gates > & - & & 6 & 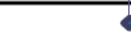 & & 4 & 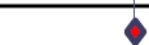 & 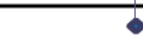 & 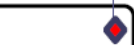 \\
\hline
\end{tabular}

\section{RESULTADOS Y DISCUSIÓN}

\subsection{Caracterización de las empresas}

Las empresas analizadas están localizadas en la Región 3 Nodo Santa Fe, según regionalización del Estado Provincial.

En relación al tamaño de las organizaciones, según la clasificación de la Secretaría de Emprendedores y de la Pequeña y Mediana Empresa de la República Argentina, las empresas A, B, C, D, E, H, se clasifican como Grandes Empresas, I, K y L como Medianas, mientras que $F, G$ y J como Pequeñas.

Las empresas A, B, C y D pertenecen al sector lácteo y ofrecen diferentes líneas de productos (la empresa A cuenta con 207 productos, B con 59, C con 160 y la empresa D con 20, incluyendo las diferentes presentaciones). Las actividades de comercialización de $\mathrm{D}$ se definen dentro del país, mientras que A, B y C cubren el territorio nacional e internacional.

Las empresas E, F, G y H pertenecen al sector de insumos, fundamentalmente alimenticios, destinados a diversas industrias: láctea, golosinas, bebidas, panificación, carnes, etc., e incluso pueden abarcar otras industrias como cosmética, fotografía, entre otras. La empresa E se dedica a la producción de preparados de fruta, la empresa $\mathrm{F}$ a proveer soluciones basadas en gelatinas e hidrolizados, $\mathrm{G}$ a la elaboración de conservas de tomate y fruta abrillantada, y $\mathrm{H} \mathrm{a}$ la producción de deshidratados y congelados de origen vegetal.

Las empresas I, J y K pertenecen al sector de carnes. I elabora fiambres, embutidos y cortes de cerdo para la venta al público y abastece a zonas regionales. Las actividades de la empresa J se basan en la cría porcina de genética y en la elaboración de numerosos productos (agrupados en Fiambres, Productos Elaborados y Cortes Frescos), y son comercializados mediante franquicias localizadas en la ciudad de Santa Fe. La empresa K se dedica a la elaboración, fraccionamiento y envasado de diferentes productos derivados del pollo.

En la Figura 1 se realiza una comparación entre el Modelo de Penso (2003) y las prácticas del PDP llevadas a cabo por cada una de las empresas analizadas. 
Figura 1. Modelo de Penso (2003) y las prácticas del PDP de las empresas analizadas.

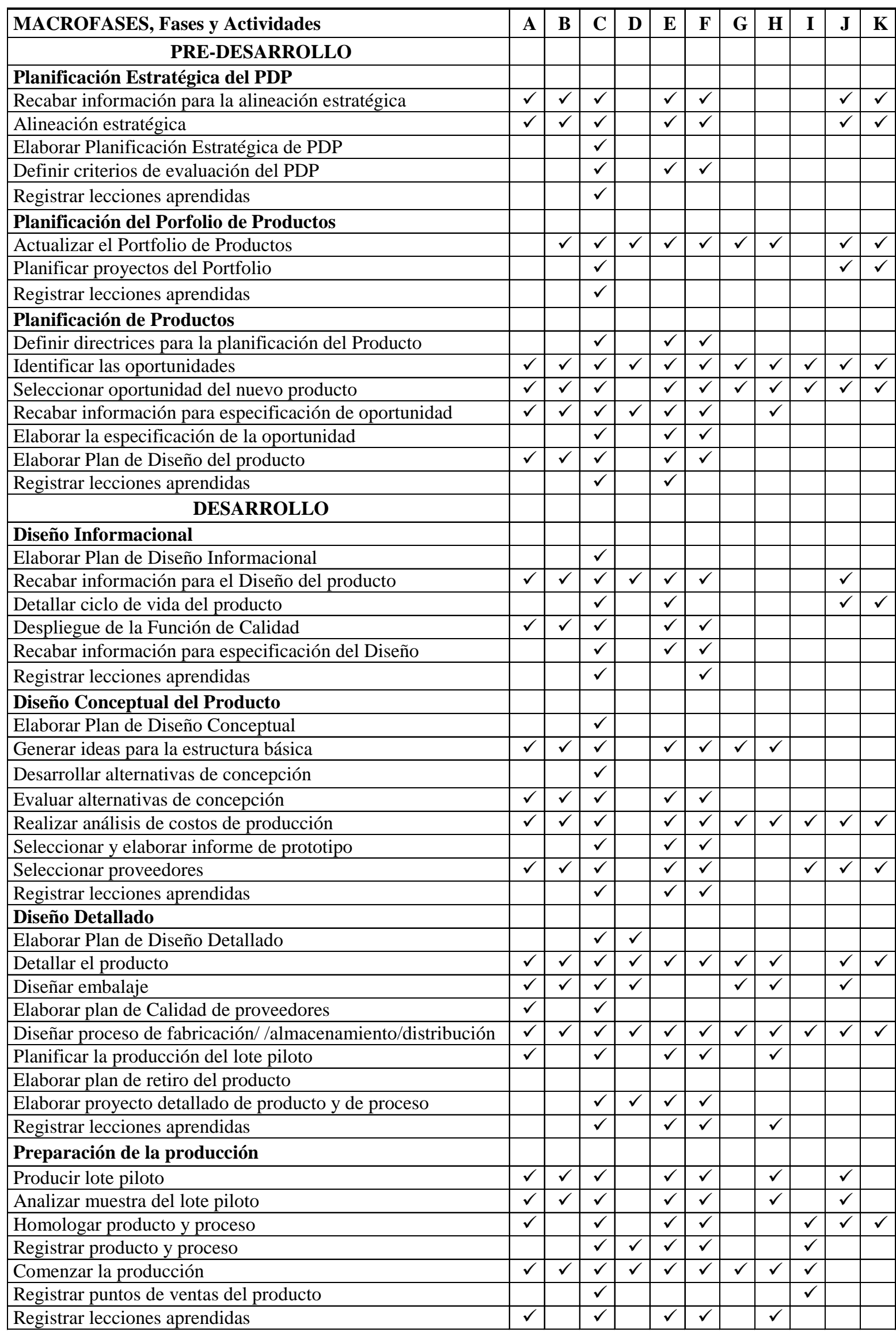


Figura 1 (cont). Modelo de Penso (2003) y las prácticas del PDP de las empresas analizadas.

\begin{tabular}{|l|c|c|c|c|c|c|c|c|c|c|c|}
\hline MACROFASES, Fases y Actividades & A & B & C & D & E & F & G & H & I & J & K \\
\hline Lanzamiento & & & & & & & & & & & \\
\hline Detallar Servicios de Atención al Consumidor & & & & & & & & & & & \\
\hline Preparar material publicitario & & & & $\checkmark$ & & & & & $\checkmark$ & & \\
\hline Implementar estrategias de lanzamiento & $\checkmark$ & $\checkmark$ & $\checkmark$ & $\checkmark$ & & & & $\checkmark$ & $\checkmark$ & & \\
\hline Registrar lecciones aprendidas & & & $\checkmark$ & & & $\checkmark$ & & & & & \\
\hline \multicolumn{1}{|c|}{ POST-DESARROLLO } & & & & & & & & & & & \\
\hline Acompañamiento del producto & & & & & & & & & & & \\
\hline Realizar auditoría post-proyecto & $\checkmark$ & & $\checkmark$ & & & & & & & & \\
\hline Evaluar satisfacción de los clientes & $\checkmark$ & $\checkmark$ & & & & & & & $\checkmark$ & $\checkmark$ & $\checkmark$ \\
\hline Monitorear el desempeño del producto & $\checkmark$ & & $\checkmark$ & $\checkmark$ & & & $\checkmark$ & $\checkmark$ & $\checkmark$ & $\checkmark$ & $\checkmark$ \\
\hline Planificar modificaciones para mejoras & $\checkmark$ & & & $\checkmark$ & & & & & & & \\
\hline Registrar lecciones aprendidas & & & & & & & $\checkmark$ & & & & \\
\hline Retiro del producto & & & & & & & & & & & \\
\hline Implementar plan de retiro del producto & & & & & & & & & & & \\
\hline Evaluar resultado económico-financiero & $\checkmark$ & & $\checkmark$ & & $\checkmark$ & $\checkmark$ & & $\checkmark$ & & $\checkmark$ & \\
\hline Registrar lecciones aprendidas & & & & & & & & & & & \\
\hline
\end{tabular}

\subsection{Macrofase Pre-Desarrollo}

En el sector lácteo, a excepción de D, las empresas reconocen la importancia de alinear la planificación del PDP con la planificación estratégica de la empresa. Por ello, la mayoría de las veces, los lanzamientos o re-lanzamientos de productos se corresponden con decisiones estratégicas relacionadas a posicionar productos, ampliar mercados, proteger posiciones de liderazgo, entre otras.

Las ideas de nuevos productos surgen de diversas fuentes: investigaciones de mercado, integrantes de la empresa, distribuidores y puntos de ventas, competencia y análisis de la cartera de productos de la empresa. Cabe destacar que A y B contratan el servicio de Investigación de Mercado a Consultoras especializadas, mientras que $\mathrm{C}$ realiza internamente esta actividad, dado que considera primordial tratar la información que surge de este tipo de estudios en forma confidencial. En el caso de D, las ideas surgen fundamentalmente del gerente general.

Los potenciales desarrollos son guiados, en A y C, por el Departamento de Marketing, y en B por el Departamento I+D, que son los encargados de monitorear el avance de PDP, conformando equipos de trabajo y designando su líder de acuerdo a las características de cada proyecto, facilitando información pertinente, coordinando reuniones periódicas y efectuando un adecuado seguimiento.

En el sector de Insumos, existen notorias diferencias en cuanto a las empresas entrevistadas. Un grupo de empresas, E y F, reconocen la importancia de alinear la planificación del PDP con la planificación estratégica de la empresa: en un gran porcentaje, los lanzamientos o relanzamientos de productos se corresponden a solicitudes específicas de clientes, y por ello las empresas consideran necesario darles respuesta en la medida en que guarden relación con las estrategias corporativas. En el caso de $\mathrm{G}$ y $\mathrm{H}$, el desarrollo de productos es esporádico, y corresponde a decisiones de la empresa, sin una vinculación directa con el cliente.

De esta manera, las ideas de nuevos productos surgen, en el primer grupo de empresas, fundamentalmente de requerimientos puntuales de clientes, y también de manera proactiva. En el primer caso, los proyectos se caracterizan por ser únicos y diferenciados, dado que 
responden a necesidades específicas de cada cliente ("productos a medida"). En el segundo caso, resultan a partir de conferencias, ferias mundiales, revistas de innovación, competidores, nuevas tendencias, reuniones anuales de las filiales de la empresa. En el segundo grupo de empresas, las ideas surgen fundamentalmente a partir de la imitación de los competidores o para aprovechar capacidad ociosa de la planta.

Los potenciales desarrollos son guiados, en E y F, por el Departamento I+D, encargado de monitorear el avance de PDP. En E, se genera un Brief (expediente) que contiene datos específicos del proyecto (objetivos, consumidores, fechas, formulación, ventas, costos) y el documento Análisis de riesgo, donde se evalúa el impacto potencial del proyecto. Todos los desarrollos son conducidos por un mismo equipo de trabajo, conformado por integrantes de diferentes áreas. La empresa $\mathrm{F}$ posee diferentes mecanismos de gestión del PDP según el tipo de proyecto (PMO o Workrequest): en los PMO (proyectos de gran magnitud, innovadores para la empresa), se realiza el Project Proposal (Propuesta de Proyecto) donde se define la idea, objetivos, alcance, estudio preliminar de costos y beneficios, requisitos funcionales y de desempeño, informaciones de proyectos anteriores y un plan de hitos; también se detallan los potenciales riesgos sobre Seguridad Alimentaria, Salud y Seguridad ocupacional y Aspectos Ambientales. De acuerdo a las características del proyecto, se selecciona un Project Manager (Líder) quien guía al equipo de trabajo, diferente para cada proyecto. Para los Workrequest (desarrollos de menor magnitud, menor innovación, y rápida ejecución), se realiza un Workplan (Plan de Trabajo) y las tareas se simplifican.

En el sector de carnes, las empresas no cuentan con mecanismos formales de planificación estratégica. En general, las ideas de nuevos productos o modificaciones de los ya existentes surgen de los directivos de la empresa, como copia de productos de la competencia y/o para lograr un mejor aprovechamiento de los desperdicios.

\subsection{Macrofase Desarrollo}

En las organizaciones del sector lácteo, en el caso de $\mathrm{A}, \mathrm{B}$ y $\mathrm{C}$ el equipo que guía el PDP delega las actividades en el área encargada del desarrollo, que según la empresa recibe diferentes denominaciones (Departamento de Desarrollo, Departamento de I+D y Departamento de Calidad). Existen diferencias entre las empresas en función de las actividades realizadas en el Desarrollo, no sólo en lo que respecta a la cantidad de tareas sino también a su profundidad. En la empresa B el proceso se orienta a lograr muestras piloto satisfactorias por medio de "prueba y error"; en las empresas A y C evidencia un mayor grado de detalle en tareas vinculadas con el diseño informacional, diseño conceptual y diseño detallado; mientras que en D no existe un área específica encargada del desarrollo.

En relación a los análisis de laboratorio, las empresas ponen de relieve la ejecución de Análisis Fisicoquímicos y Microbiológicos y de Determinación de Vida Útil mediante métodos que permiten garantizar la calidad de los productos, como así también de Análisis Sensoriales con el objeto de lograr la aceptación del consumidor. Las empresas A, B y C realizan internamente estos análisis, mientras que la empresa $\mathrm{D}$ encarga parte de los estudios a laboratorios externos y no cuenta con un personal especializado para los Análisis Sensoriales.

En cuanto a la documentación de la información, una actividad clave de esta macrofase, las empresas reconocen la importancia de los registros, pero existen diferencias sustanciales en su aplicación. En la empresa C la documentación es un hábito, y no es posible avanzar en el desarrollo de un proyecto si no se presentan una serie de informes (Ficha Especificaciones del Producto, Ficha de Proceso y Formulación, Ficha de Proveedores, entre otras). La empresa A cuenta con una Ficha de Producto, donde se registran de forma unificada todas las especificaciones de cada proyecto de desarrollo, resulte o no en un lanzamiento efectivo de 
producto. La empresa $\mathrm{B}$ documenta cada proyecto únicamente cuando ha cumplido un análisis productivo y económico positivo. En $\mathrm{D}$, la documentación no es un hábito.

En el sector de insumos, las actividades de esta macrofase, en el caso de E y F, son las de mayor nivel de estandarización. El PDP continúa guiado por el Departamento de Investigación y Desarrollo en las áreas de laboratorio, donde se efectúan muestras y comienza una serie de loops (retroalimentaciones) con el cliente hasta lograr resultados satisfactorios. Luego se realiza un escalamiento para ensayar la producción en serie del insumo, y, si el cliente manifiesta satisfacción con el resultado de la prueba industrial, se firma un documento que valida el proceso y el resultado logrado, incorporando formalmente el insumo a la planificación de la producción de la empresa. Uno de los puntos críticos es la realización de pruebas de laboratorio, para lo cual se elaboran Planes de trabajo que contienen información detallada de las formulaciones esperadas, los análisis a realizar y el cronograma de trabajo. En el caso de $\mathrm{G} \mathrm{y} \mathrm{H}$, el proceso de desarrollo no sigue un lineamiento previamente definido. Se comienzan con la búsqueda de una formulación adecuada, que cumpla con los reglamentos legales, a partir de laboratorios propios, siendo necesario en algunos casos la participación de laboratorios externos. El Análisis Sensorial de productos está a cargo de personal de la empresa y familiares, sin contar con paneles especializados.

En cuanto a la documentación de la información, las empresas reconocen la importancia de los registros, pero existen algunas diferencias en su aplicación. En la empresa F la documentación es un hábito, y no es posible avanzar en el desarrollo de un proyecto si no se presentan todos los informes pertinentes (Project Charter, Project Development and Transfer, entre otros). La empresa E cuenta con un Brief y una Hoja de proceso, donde se registran de forma unificada todas las especificaciones de cada proyecto sólo cuando han cumplido un análisis productivo y económico positivo. $\mathrm{G}$ y $\mathrm{H}$ no tienen el hábito de documentar los procesos, limitándose a registrar las formulaciones exitosas.

En lo que respecta al sector de carnes, la empresa I lleva a cabo el desarrollo de manera menos estructurada; una vez que se selecciona la idea de producto, no se sigue una metodología específica, sino que se intenta materializar el producto a partir de "prueba y error", y, de ser necesario, se contrata un laboratorio externo. En las empresas J y K, el proceso de desarrollo del producto tampoco sigue un lineamiento previamente definido; no obstante es llevado a cabo por un equipo donde participan el responsable de planta (encargado de realizar ensayos básicos en laboratorio, y en caso de necesitar, solicitar asesoramiento externo) y responsables de Ventas y Administración, y se realizan reuniones periódicas para revisar el avance del desarrollo. Los Análisis Sensoriales son realizados por personal de la empresa y personas allegadas, sin contar con paneles especializados. Las tres empresas manifiestan la escasa sistematización de registros, limitándose a documentar las formulaciones exitosas.

\subsection{Macrofase Post-Desarrollo}

En esta macrofase, las empresas del sector lácteo $\mathrm{A}, \mathrm{B}$ y $\mathrm{C}$ manifestaron realizar el seguimiento de los productos en el mercado, comparando las proyecciones elaboradas con los resultados obtenidos, $y$, en base a esta información, la gerencia toma la decisión de la continuidad o del retiro del producto. D no efectúa un seguimiento formal, siendo fundamentalmente las situaciones de quejas o devoluciones de producto las que obligan a la empresa a revisar sus estrategias. Cabe mencionar que ninguna de las empresas analizadas elabora un plan de retiro de productos, sino que la decisión se toma en función del grado de aceptación de los mismos en el mercado. Por último, A, B y C manifestaron la importancia de aplicar la Trazabilidad en sus productos, para poder realizar un adecuado seguimiento de los mismos y proceder a retirar un determinado lote en caso de un eventual incidente. 
En el sector de insumos se observa que esta Macrofase es la de menor sistematización del PDP. Las empresas E y F no manifiestan llevar a cabo un seguimiento de los productos en el mercado; el seguimiento se basa en la comparación de las proyecciones elaboradas con los resultados obtenidos. Las actividades realizadas en esta Macrofase se orientan fundamentalmente al Retiro de productos en caso de que ocurra alguna eventualidad en relación a los mismos (cabe destacar que el producto final de las empresas analizadas supone productos intermedios para sus clientes, quienes son los que se dirigen al mercado de consumidores finales; por ello, según en qué instancia se detecte el problema, las empresas $\mathrm{E}$ y $\mathrm{F}$ asumen la responsabilidad y los costos de retirar la producción). En este sentido, las empresas reconocen la importancia de aplicar la Trazabilidad, para poder realizar un adecuado seguimiento de los productos y proceder a retirar un determinado lote en caso de incidentes. La empresa $\mathrm{G}$ efectúa el seguimiento del producto a partir de Registros de no conformidad del cliente, para poder mejorar los siguientes lotes de producción. En la empresa $\mathrm{H}$, el seguimiento se realiza a partir de la comparación de los pronósticos de venta y los montos reales, y con esa información se decide la continuidad, modificación o retiro del producto.

En el sector de carnes, la empresa I analiza si las ventas del producto generan ganancias y en ese caso se continúa su producción. En J el seguimiento de los productos es la única fase que presenta registros sistemáticos: se realiza de manera cualitativa (opinión del cliente) como también de manera cuantitativa (nivel de ventas y rentabilidad), y con esa información se toma la decisión de retirar el producto del mercado u ofrecerlo de forma diferente. La empresa $\mathrm{K}$, en las ocasiones en las que realiza desarrollo de productos, aplica actividades del seguimiento de los mismos.

\section{CONCLUSIONES}

El objetivo del presente trabajo ha sido estudiar la Gestión del Proceso de Desarrollo de Productos en empresas productoras de alimentos de la Provincia de Santa Fe. Se utilizó como referencia el Modelo de Penso para comparar las prácticas relacionadas con el PDP llevadas a cabo por las empresas bajo estudio.

Entre las principales conclusiones se evidencia que solo algunas de las empresas disponen de metodologías y prácticas internalizadas para la Gestión del PDP. Además, existen notorias diferencias en relación a los niveles de detalle que presenta la sistematización de las actividades que involucra el PDP. Se observa un grupo de empresas, A, B, C, E y F (empresas medianas y grandes de los sectores lácteo e insumos) que disponen de un modelo de Gestión de PDP definido y que las metodologías y prácticas del PDP se encuentran internalizadas para su aplicación sistemática; mientras que otro grupo, compuesto por D (empresa mediana del sector lácteo), G, H (empresas pequeña y mediana del sector insumos) y I, J y K (empresas pequeñas y grande del sector carnes), poseen escasa sistematización en la Gestión del PDP. Ello permitiría concluir que las diferencias pueden observarse, fundamentalmente, en relación al tamaño de las organizaciones (a mayor tamaño, mayor grado de sistematización del PDP). Por otro lado, la información indica que, a partir de la comparación entre diferentes sectores, el sector carnes presenta menor nivel de desarrollo del PDP. Se destaca que el presente trabajo presenta resultados preliminares, y sería necesario el análisis de un número mayor de empresas para poder ser concluyente en este sentido.

En lo que respecta al Pre-Desarrollo, el primer grupo de empresas se focaliza en actualizar su portfolio en función de las decisiones estratégicas adoptadas y del mercado en el que se insertan, lo que les permite identificar las oportunidades del contexto, seleccionar nuevos productos y definir directrices para su planificación. Sin embargo, no se evidencia una adecuada sistematización y registro de las decisiones de estas fases ni de lecciones 
aprendidas. En el segundo grupo de empresas las actividades de esta macrofase son las que se evidencian más ausentes: en general, intentan adecuar su capacidad técnica y productiva a las oportunidades de mercado que se presentan, sin tener en cuenta lineamientos estratégicos dado que no poseen sistemas de planificación formales. En general, este grupo se reconoce como seguidoras, buscando instalarse en los mercados desarrollados por los grandes competidores a partir de alguna variable de diferenciación. Debido a la naturaleza del sector alimenticio, las fases que componen la macrofase de Desarrollo son las más consolidadas en todas las empresas. Entre las que componen el primer grupo, se observa un profundo nivel de detalle en la sistematización de las actividades de esta macrofase, evidenciado en la cantidad de documentación utilizada, puntos de control y validaciones requeridas. En el segundo grupo, las actividades de desarrollo se reconocen fundamentales, aunque se realizan con menor grado de detalle. Finalmente, la macrofase de Post-Desarrollo demuestra ser la de menor sistematización en todas las empresas analizadas.

La utilización de un modelo referencial para el PDP auxilia, organiza y direcciona el desarrollo de nuevos productos. El Modelo de Penso resultó de utilidad para analizar las empresas estudiadas, dado que presenta un nivel adecuado de detalle que permite la comparación del PDP. En relación a la propuesta de la autora, las empresas del primer grupo podrían fortalecer las actividades de las macrofases Pre-desarrollo y Post-desarrollo, mientras que las empresas del segundo grupo deberían comenzar con la aplicación de intervenciones en la gestión del PDP de forma de conseguir mejoras parciales e incrementales.

\section{REFERENCIAS}

ABU, N. et al. The pre-development process implementation of product innovation: A Malaysian food and beverage manufacturing SMEs survey. International Journal of Business and Management Science, v. 5, n. 1, p. 39-49, 2012.

EARLE, M. D. Changes in the food product development process. Trends in Food Science \& Tecnology. vol. 8, Pp. 19-24. Cambridge, 1997.

FULLER, G. W. New food product development: from concept to marketplace. CRC Press, Florida, 1994.

GRAF, E. Y SAGUY, I. Food Product Development: from Concept to the Marketplace. Avi, New York, 1991.

HOLMAN, R. El Futuro del Desarrollo de Productos, Gestión, vol. 9, nº 2, 2004.

LERMA KIRCHNER, A. Desarrollo de nuevos productos, una visión integral. Cuarta edición. Querétaro, México: Cengage Learning, 2010.

PENSO, C. Modelo de Referência para o Processo de Desenvolvimento de Produtos na Indústria de Alimentos. Tese de Mestrado, Universidad Federal de Santa Catarina, Florianópolis, Brasil, 2003.

ROZENFELD, H. et al. Gestão de Desenvolvimento de Produtos: uma referência para a melhoria do processo. São Paulo: Saraiva, 2006.

SAUTU, R; BONIOLO, P; DALLEY, P. Y ELBERT, R. Manual de Metodología. Construcción del Marco Teórico, Formulación de los Objetivos y Elección de la Metodologia, CLACSO, Buenos Aires, 2005.

\section{AGRADECIMIENTOS}

Los autores agradecen la contribución económica de la Agencia Nacional de Promoción Científica y Tecnológica (PICT 2015-1629) y de la UNL (CAI+D 2016 PI 50120150100216LI). 